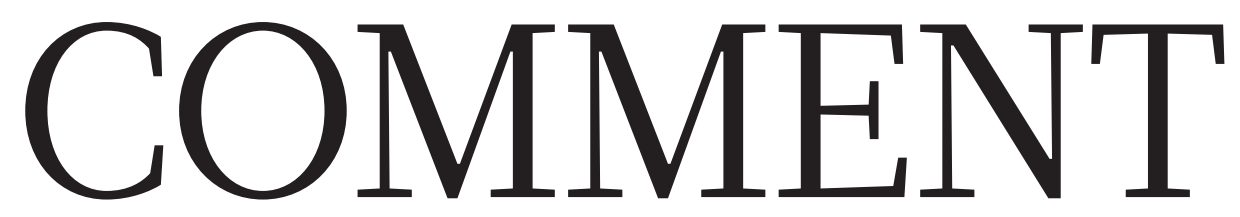

BIBLIOMETRICS Women's

research output and citations analysed $\mathbf{p . 2 1 1}$
ANIMATION Gollum

co-creator on digital storytelling $\mathbf{p . 2 1 4}$
doCUMENTARY Celebrating

John Milne, founder of modern seismology $\mathbf{p . 2 1 6}$
AGRICULTURE Funds needed to fight banana fungus ravaging southeast Asia p.218

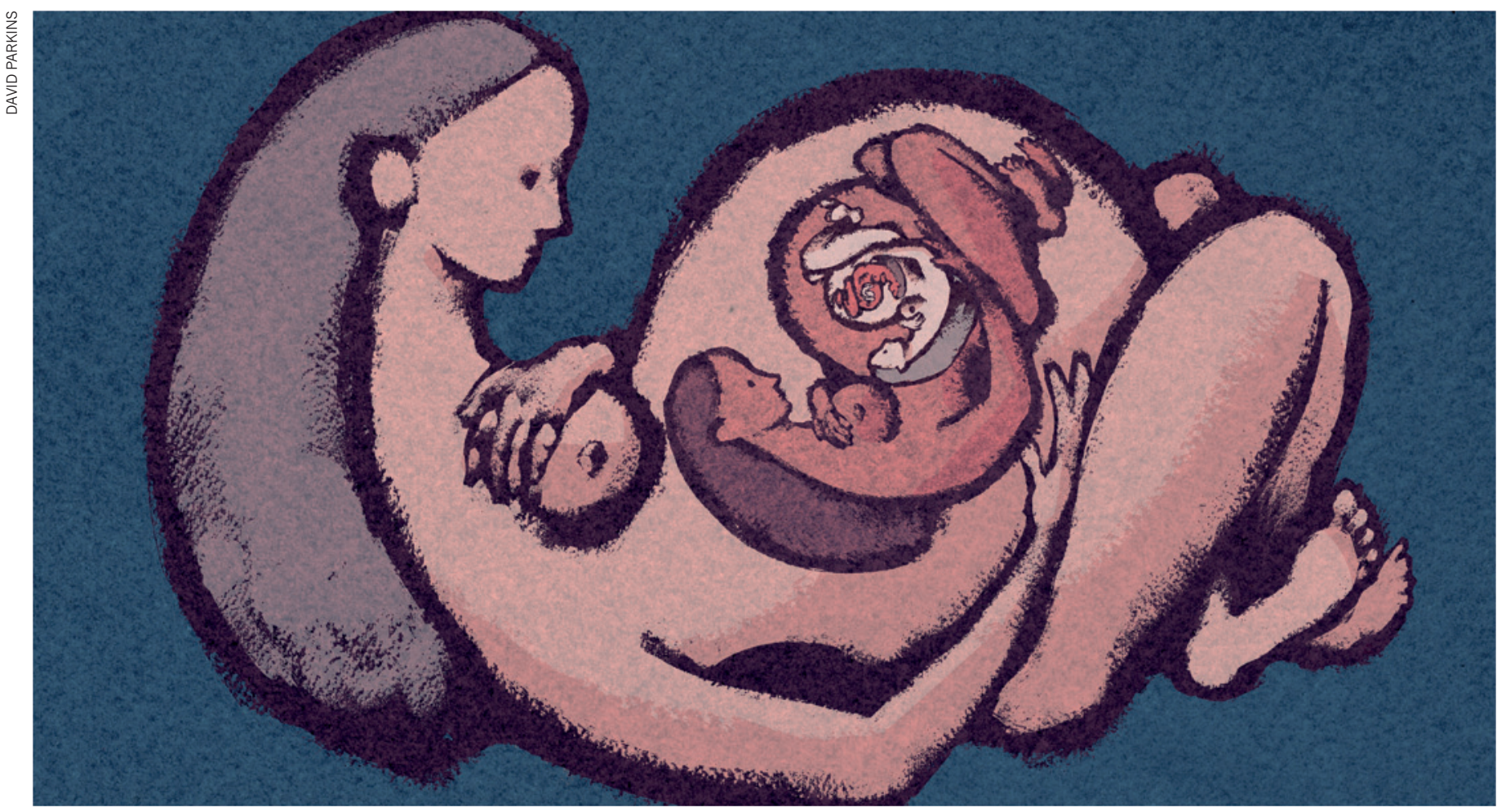

\title{
Support mothers to secure future public health
}

Evidence that long-term health is shaped by the environment in early life calls for prenatal interventions to tackle chronic disease, argue David Barker and colleagues.

$\mathrm{W}$ orldwide, chronic diseases are on the rise. Globally, the prevalence of adult-onset diabetes is expected $^{1}$ to approximately double by 2030 , and that of cardiovascular disease to increase by about $35 \%$ (ref. 2). Current approaches to curbing this situation are failing.

More than 30 years of epidemiological studies using data from several hundred thousand people around the world point to factors during prenatal and early childhood development that contribute to these statistics.

Together, these data suggest that instead of focusing exclusively on people's genes, or on their diets and lifestyles in adulthood, we need a developmental approach to public health. People working in public health must support girls and young women with low incomes to help them to feel more in control of their lives and so better able to prioritize healthy eating. At the same time, better access to quality food is necessary so that it is easier for people to make healthier choices. This would improve mothers' nutrition and thereby the health of future generations.

Clues that chronic disease might result from perturbations in growth during early life came from an unusual data source.
Nearly 30 years ago, an exhaustive search of every record office in every town and city in England and Wales unearthed ledgers in the county archive of Hertfordshire containing health visitors' records for thousands of babies born between 1911 and 1948. These records documented the babies' weight at birth and at one year old, whether they were fed breast milk or formula, the age at which they were weaned, and significant health events, such as bouts of illness, in their early life.

Tracing many of the individuals named in these records revealed that their health in adulthood was associated with how they $>$ 
b had grown in the womb. The Hertfordshire data and similar records from other UK towns revealed, for instance, that a person weighing 2.7 kilograms ( 6 pounds) at birth has a $25 \%$ higher risk of contracting heart disease in later life, and a 30\% higher risk of having a stroke, compared with someone weighing 4.1 kilograms ( 9 pounds) at birth ${ }^{3}$.

These findings were soon strengthened by data from a cohort of 20,000 people born in Helsinki between 1924 and 1944. This study showed, for example, that if all the babies at birth had had weights within the highest third of the total range, the incidence of diabetes in later life would have been halved ${ }^{4}$. In the years since, numerous other studies, involving people from places as diverse as Europe, India, Guatemala, the Philippines and South Africa, have revealed similar correlations with effects that extend to the health of grandchildren.

\section{DEVELOPMENT AND DISEASE}

In the past 15 years, researchers have begun to understand the biology underlying the links between development and chronic disease. The evidence suggests that women should start eating healthily well before they get pregnant. Women who are obese, for example, accumulate more metabolites (such as insulin, lactate and triglycerides) in their ovarian follicles ${ }^{5}$ than do women who are not obese. This accumulation can reduce their fertility and increase the likelihood that their offspring will develop certain diseases, such as diabetes, cardiovascular disease or cancer, later in life.

At the moment of conception, the growing embryo seems to be exquisitely sensitive to its nutritional environment. Studies of babies born through in vitro fertilization, for instance, have shown that birth weights can be affected simply by changing the constituents of the medium in which the embryos are cultured.

After conception, if resources are in short supply, investment in low-priority organs, such as the kidneys and lungs, which do not function in the womb, may be traded off to protect more important ones such as the brain. Studies in rats and piglets, as well as research using ultrasound to track the growth of organs in human fetuses, show that adverse conditions in the womb are associated with smaller kidneys with fewer nephrons, which regulate the concentration of water and soluble substances in the blood. Fewer nephrons means more wear and tear to existing nephrons, more nephron loss and an increase in blood pressure with age.

A mother's stored nutrients and the turnover of protein and fat in her tissues - a reflection of her lifetime nutrition - seem to be particularly important in later fetal life. Studies of mice, rats and pregnant women have shown that mothers with a greater

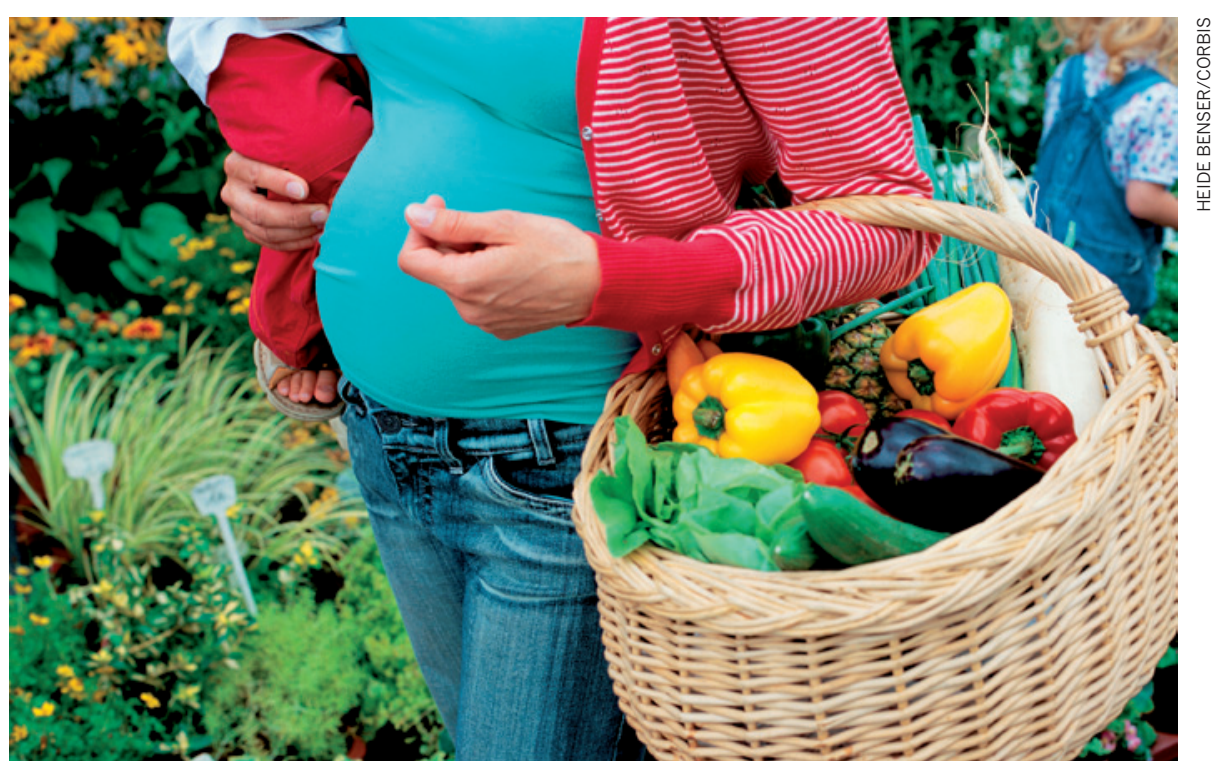

The effects of healthy eating before and during pregnancy are far-reaching.

muscle-to-fat ratio and larger organs have a higher rate of protein turnover. This enables them to make amino acids more readily available to their fetuses, especially during the later weeks of pregnancy, when nutritional demands are greatest.

Differences in the size and shape of the placenta may also reflect a mother's nutritional status and serve as biomarkers of a fetus's early experience. These observations come mainly from work on animals, but also from cohort studies relating measurements of mothers' placentas at the time of birth to their diets and the birth weights of their babies. Individuals, who as fetuses were nourished by placentas that are long for their breadth, for example, are up to twice as likely to develop colorectal cancer and, if a first child, up to three times as likely to develop coronary heart disease compared with those who had been nourished by placentas of average size and shape ${ }^{6}$.

And although babies deprived in utero but then born into conditions of plenty often 'catch up' in body weight in their first year of life, such compensatory growth has costs. In fish and birds, offspring who start small and grow fast have fewer offspring, are more susceptible to infections and disease, and live shorter lives than offspring that start life larger. The Helsinki data similarly indicate that boys who catch up between birth and age seven do not live as long as those whose growth follows a more standard trajectory.

\section{ACHIEVING CHANGE}

Today, millions of fetuses are receiving inadequate nutrition because their mothers are starving, malnourished or overweight. To understand the factors driving poor nutrition, we have been running focus groups and surveys with mothers for the past decade - first, in Southampton, UK, beginning in 2004, and then in Klamath Falls, Oregon, starting in 2007.

By asking questions to try to understand what healthy eating means to mothers, and how they would ideally like to feed their children, we have established that young women know that they and their children should eat a balanced and varied $\operatorname{diet}^{7}$. But other challenges, such as having to balance the cost of food with the potential for waste, leave mothers feeling out of control and with little motivation for the battle involved in creating good eating habits in their families.

Under these circumstances, the publichealth approach currently used across industrialized nations, of providing women with information about healthy eating, seems unlikely to be effective. Our work and that of others strongly suggests that a better strategy would be to support women to identify the barriers they face and, where possible, to empower them to generate their own solutions.

Ten years ago, a team of local and international health-care researchers worked with women from villages in Nepal, aiming to reduce the high levels of mortality among them and their newborn babies ${ }^{8}$. Women were encouraged to join discussion groups run by a facilitator who helped them to formulate simple strategies to improve perinatal care that would work in their communities - such as the production and distribution of basic, home-birth delivery kits. Over the following two years, the $8 \%$ of the 28,000 village women who attended the meetings spread the messages to others and, solely because of this intervention, infant mortality fell by $30 \%$ and maternal mortality by $80 \%$.

A similar empowerment mechanism underpins the 'disease-self-management' programmes that are increasingly being used all over the world, often with striking effects ${ }^{9}$. 
In these, people with chronic diseases are brought together to support each other to better manage their conditions.

Since 2009, we have been involved in the Southampton Initiative for Health, which uses an empowering, problem-solving approach to improve the diets and physical activity levels of Southampton's most disadvantaged young women and their children. The programme has involved training the staff of the city's Sure Start Children's Centres - providers of services such as baby clinics, breastfeeding and weaning support, dentistry, parenting and cookery classes - in having conversations that encourage women to identify problems and generate solutions to change behaviour.

Although the data suggest that attending centres staffed by workers using this approach enhances women's sense of empowerment, to improve their nutritional status we need both to help women to feel more in control of their food choices and to make it easier for them to make better choices.

On a small scale, such a multilevel approach has proved effective. Trials in Canada, Australia and the United States demonstrate that the diets of small-town residents can be improved when efforts to enhance people's sense of empowerment in relation to healthy eating are pursued alongside local media campaigns to promote the benefits of eating well, together with programmes that help people to gain better access to fruits and vegetables and skills in food preparation. The challenge is to scale up such efforts to the wider public-health arena, because this means engaging political and commercial interests,

including those of powerful food companies.

We believe that the methods used by people working in public health to engage politicians and food companies need to undergo a similar transformation to those being used to engage individuals. So far,

\section{"We need to} help women to feel more in control of their food choices." public-health advocates have called for regulation and legislation as means to improve diets - an increased tax on fatty and sugary foods, for instance. Yet this is unlikely to happen because raising the tax on soft drinks, say, is not in the interests of industry, or of politicians, who are sensitive to industry pressures and to a public desire for cheap soft drinks.

Instead of wagging fingers, we need to generate consensus. Empowering consumers to call for better access to better food will put pressure on politicians to respond to voters, and on the food industry to please their customers.

More than 20 years ago, one of us (D.B.) wrote $^{10}$ in this journal that "if more was known about the processes by which the environment in early life influences adult health ... the rise in incidence of 'Western' disease [might be] minimized." Today, we have the knowledge to readily prevent chronic diseases, had we but the will to do so.

David Barker died on 27 August 2013, after writing the first draft of this Comment (see Nature 502, 304; 2013). Before his death, he was professor of clinical epidemiology at the Medical Research Council (MRC) Lifecourse Epidemiology Unit, University of Southampton, UK; professor of cardiovascular medicine at the Heart Research Center, Oregon Health and Science University in Portland; and visiting professor at the Center for the Study of Human Health, Emory University, Atlanta, Georgia. Mary Barker is senior lecturer in psychology at the MRC Lifecourse Epidemiology Unit, University of Southampton, UK. Tom Fleming is professor of developmental biology at the Centre for Biological Sciences, University of Southampton, UK. Michelle Lampl is director of the Center for the Study of Human Health and Professor of Anthropology at Emory University, Atlanta, Georgia.

e-mail:meb@mrc.soton.ac.uk

1. Chen, L., Magliano, D. J. \& Zimmet, P. Z. Nature Rev. Endocrinol. 8, 228-236 (2012).

2. Laslett, L. J. et al. J. Am. Coll. Cardiol. 60, S1-S49 (2012).

3. Barker, D. J. P. Brit. Med. Bull. 53, 96-108 (1997).

4. Barker, D. J. P., Eriksson, J. G., Forsén, T. \& Osmond, C. Int. J. Epidemiol. 31, 1235-1239 (2002).

5. Robker, R. L. et al. J. Clin. Endocrinol. Metab. 94, 1533-1540 (2009).

6. Barker, D. J. P. \& Thornburg, K. L. Placenta $\mathbf{3 4 ,}$ 841-845 (2013)

7. Barker, M. et al. Pub. Health Nutr. 11, 1229-1237 (2008).

8. Manandhar, D. S. et al. Lancet 364, 970-979 (2004).

9. Bodenheimer, T., Lorig, K., Holman, H. \& Grumbach, K. J. Am. Med. Assoc. 288, 2469 2475 (2002)

10.Barker, D. J. P. Nature 338, 371-372 (1989).

\section{Global gender disparities in science}

\section{Cassidy R. Sugimoto and colleagues present a bibliometric analysis confirming that gender imbalances persist in research output worldwide.}

$\mathrm{D}$ espite many good intentions and initiatives, gender inequality is still rife in science. Although there are more female than male undergraduate and graduate students in many countries ${ }^{1}$, there are relatively few female full professors, and gender inequalities in hiring ${ }^{2}$, earnings ${ }^{3}$, funding ${ }^{4}$, satisfaction ${ }^{5}$ and patenting ${ }^{6}$ persist.

One focus of previous research has been the 'productivity puzzle'. Men publish more papers, on average, than women ${ }^{7}$, although the gap differs between fields and subfields. Women publish significantly fewer papers in areas in which research is expensive ${ }^{8}$, such as high-energy physics, possibly as a result of policies and procedures relating to funding allocations ${ }^{4}$. Women are less likely to participate in collaborations that lead to publication and are much less likely to be listed as either first or last author on a paper ${ }^{7}$. There is no consensus on the reasons for these gender differences in research output and collaboration - whether it is down to bias,

\section{DNATURE.COM See Nature's special issue on women in science: nature.com/women}

childbearing and rearing ${ }^{9}$, or other variables. It has been suggested that what women lack in research output they make up for in citations, particularly in fields with 'greater career risk ${ }^{8}$ - that is, fields with long lags between doctoral education and securing a faculty position, such as ecology. But again, there is no consensus on the relative impact of women's work compared to men's.

The present state of quantitative knowledge of gender disparities in science has been shaped primarily by anecdotal reports and studies that are highly localized, 